\title{
Rotating Consensus of LFMAS in an Elliptical Orbit with Switching Topology
}

\author{
Gun Li \\ School of Aeronautics and Astronautics \\ University of Electronic Science and Technology of China \\ ChengDu, China \\ ligun@uestc.edu.cn
}

\author{
Meng-jie Niu \\ School of Aeronautics and Astronautics \\ University of Electronic Science and Technology of China \\ ChengDu, China \\ mengjieniu@163.com
}

\begin{abstract}
This paper is devoted to the study of rotating consensus problem for leader-following multi-agent system with and without multiple time-delays in an elliptical orbit and both switching topology which is kept connected. We first define rotating consensus problem of leader-following multi-agent system in a circular orbit, and through the mapping relationship between ellipse and circular, extend rotating consensus problem to elliptical orbit. Then, we propose two new protocols for leader-following multi-agent system with and without multiple time-delays. The main results provide sufficient conditions for existence of rotating consensus for leader-following multi-agent with and without multiple time-delays in an elliptical orbit.. Simulations are provided to show the effectiveness of the obtained theoretical results.
\end{abstract}

Keywords-rotating consensus; leade -following mult-agent; elliptical orbit; multiple time-delays; switching topology.

\section{INTRODUCTION}

Due to the technological advances in communication and computation, distributed coordination of networks of dynamic agents has attracted several researchers in many fields in recent years and many results have been obtained in [1-9]. In real systems, information interchange between each agent is often associated with time delays and the network graph should be switching as communication links in a multi-agent system may experience failures or recons- tructions. Among many of the researches, the consensus problem is one of the most widely studied issues which is that each agent updates its control input based on the information of its neighbors in such a way that all agents get to a common value in final. Leader-following multi agent consensus has been actively studied and get a series of splendid achievements [1-3]. In [1], Gang et al. investigated distributed controller design for a leader-follower network with communication delays. Hong[2] et al. was concerned with a leader-follower problem for a multi-agent system with a switching interconnection topology. In [3], Hu et al. investigated a leader-following consensus problem with time-varying coupling delays. Cooperative control for multi agent systems has been applied to formation control, mobile robots, unmanned air vehicles, satellites, etc. For example, Zhao[4] investigated the consensus problem of data-sampled multi-agent systems with Markovian switching topologies. Liu[5] studied the consensus problems of multi-agent systems on directed networks. In [6], Sepulchre et al. proposed a methodology to stabilize isolated relative equilibria in a model of identical, steered particles moving in the plane at unit speed. In [7], Pavone et al. proposed decentralized control policy for symmetric formations in multi-agent systems. Based on the work of [7], Lin[8] investigated collective rotating motions of second-order multi-agent system. Ren [9] extended the results of Pavone[10] et al. to 3D by introducing a rotating formation matrix in consensus arithmetic. In [10], YANG et al. studied distributed rotating consensus in networks of second-order agents using only local position information in three-dimensional space by extending the results of [8].

\section{GRAPH THEORY}

The communication network can be modeled through an undirected weighted graph $G=(S, e, A)$ of order $n$ with a set of nodes $S=\left(s_{1}, s_{2}, \cdots, s_{n}\right)$, and a set $e \subseteq S \times S$ of edges, and a weighted adjacency matrix $\boldsymbol{A}=\left[a_{i j}\right] \in R^{n \times n}$ with non-negative elements $a_{i j}$ for all $i, j \in I=\{1,2, \cdots, n\}$. An edge of $G$ is denoted by $e_{i j}=\left(s_{i}, s_{j}\right)$, where the first element $s_{i}$ of $e_{i j}$ is said to be the tail of the edge and the other, $s_{j}$ to be the head. Then the set of neighbors of node $s_{i}$ is denoted by $N_{i}=\left\{s_{j} \in S: a_{i j}>0\right\}$. The adjacency elements associated with the edges are positive, that is, $e_{i j} \in e \Leftrightarrow a_{i j}>0$. Moreover, we assume $a_{i i}=0$ and $a_{i j}=a_{j i}$ for all $i, j \in I$. A diagonal matrix $\boldsymbol{\Delta}=\operatorname{diag}\left[\boldsymbol{\Delta}, \boldsymbol{\Delta}_{2}, \ldots, \boldsymbol{\Delta}_{n}\right]$ is a degree matrix of $G$, whose diagonal elements $\boldsymbol{\Delta}_{i}=\sum_{j=1}^{n} a_{i j}$ for all $i=1,2, \cdots, n$.Then, the Laplacian of the undirected graph $G$ is defined as

$\boldsymbol{L}=\boldsymbol{\Delta}-\boldsymbol{A}$. An undirected path is a sequence of ordered edges of the form $\left(s_{i}, s_{k 1}\right),\left(s_{k 1}, s_{k 2}\right), \cdots,\left(s_{k l}, s_{j}\right)$, where $s_{i_{j}} \in S$ in an undirected graph. A undirected graph is called connected if there is a path from every node to every other node.

In this paper, we consider another graph $\tilde{G}$, associated with the system consisting of $n$ following agents and one leader (labeled 0). Similarly, we define a diagonal matrix 
$\boldsymbol{D} \in R^{n \times n}$ to be a leader adjacency matrix associated with $\tilde{G}$ with diagonal elements $d_{i}$, where $d_{i}=a_{i 0}$ for some constant $a_{i 0}>0$ if node 0 is a neighbor of node $i$, otherwise, $a_{i 0}=0$.

Lemma 1: If a undirected graph $G$ is strongly connected, zero is one eigenvalue of $L$, and $\boldsymbol{1}$ is the corresponding eigenvector, i.e., $\boldsymbol{L} \mathbf{1}=\mathbf{0}$, where $\mathbf{1}$ represents $[1,1, \cdots, 1]^{T}$.

Lemma 2: For a given symmetric matrix $S=\left[\begin{array}{ll}S_{11} & S_{12} \\ S_{21} & S_{22}\end{array}\right]$, where $\quad S_{11} \in R^{r \times r} \quad, \quad S_{12} \in R^{r \times(n-r)}$, $S_{21} \in R^{(n-r) \times r}, S_{22} \in R^{(n-r) \times(n-r)}$.Then $S<0$ holds if and only if $S_{11}<0, S_{22}-S_{21} S_{11}^{-1} S_{12}<0 \quad$ or $S_{22}<0$, $S_{11}-S_{12} S_{22}^{-1} S_{21}<0$.

\section{STATE DESCRIPTION}

In this paper, we considered second-order leader following multi-agent system with the graph $\tilde{G}$. Suppose the dynamics of the ith $(i \in I)$ agent is:

$$
\dot{\boldsymbol{r}}_{\boldsymbol{i}}=\boldsymbol{v}_{i}, \quad \dot{\boldsymbol{v}}_{i}=\boldsymbol{u}_{i}, i=1,2, \ldots, n
$$

where the vectors $\boldsymbol{r}_{\boldsymbol{i}}, \boldsymbol{v}_{\boldsymbol{i}} \in C \approx R^{2}$ respectively denote the position and velocity of the agent $s_{i}$ and $u_{i}(t) \in C$ is the control input. Some followers in $\tilde{G}$ are impressed by the leader and other neighbor agents, while the motion of the leader is independent.

The dynamics of the leader is described as follows:

$$
\dot{r}_{0}=v_{0}
$$

where $\boldsymbol{r}_{\boldsymbol{0}}$ are the position and $\boldsymbol{v}_{\boldsymbol{0}}$ is the desired constant velocity. So our main objective was to design rules to make all leader-following multi-agent achieve rotating consensus in an elliptical orbit. Firstly, we assumed that each agent moves in anti-clockwise direction. Before giving the main results, we needed to make the following Definition 1 in a circular orbit.

Definition 1. The leader-following multi-agent system achie -ves rotating consensus in a circular orbit if

$$
\begin{aligned}
& \lim _{t \rightarrow \infty}\left[\boldsymbol{r}_{e i}(t)-\boldsymbol{r}_{e \theta}(t)\right]=0 \\
& \lim _{t \rightarrow \infty}\left[\boldsymbol{r}_{e i}(t)-\boldsymbol{r}_{e k}(t)\right]=0 \\
& \lim _{t \rightarrow \infty}\left[\boldsymbol{v}_{e i}(t)-\boldsymbol{v}_{e \theta}(t)\right]=0 \\
& \lim _{t \rightarrow \infty}\left[\boldsymbol{v}_{e i}(t)-\boldsymbol{v}_{e \boldsymbol{k}}(t)\right]=0 \\
& \lim _{t \rightarrow \infty}\left[\dot{\boldsymbol{v}}_{e 01}(t)+\boldsymbol{j} \dot{v}_{e 02}(t)-\boldsymbol{j} \omega\left(\boldsymbol{v}_{e 01}(t)+\boldsymbol{j} \boldsymbol{v}_{e 02}(t)\right]=0\right. \\
& \lim _{t \rightarrow \infty}\left[\dot{\boldsymbol{v}}_{e i 1}(t)+\boldsymbol{j} \dot{\boldsymbol{v}}_{e i 2}(t)-\boldsymbol{j} \boldsymbol{\omega}\left(\boldsymbol{v}_{e i 1}(t)+\boldsymbol{j} \boldsymbol{v}_{e i 2}(t)\right]=0\right.
\end{aligned}
$$

For all $i, k \in I$, where $\boldsymbol{r}_{e i}=\boldsymbol{r}_{e i 1}+\boldsymbol{j} \boldsymbol{r}_{e i 2}, \boldsymbol{v}_{e i}=\boldsymbol{v}_{e i 1}$ $+j v_{e i 2}, \quad r_{e 0}=r_{e 01}+j r_{e 02}, \quad v_{e 0}=v_{e 01}+j v_{e 02}$.

Note that there exist a mapping relation between circle and ellipse as

$$
r_{e i}=\frac{r_{i 1}-c}{a}+j \frac{r_{i 2}-d}{b} \quad v_{e i}=\frac{v_{i 1}}{a}+j \frac{v_{i 2}}{b}
$$

if we denote $\boldsymbol{r}_{e i 2}=\left(\boldsymbol{r}_{i 2}-d\right) / b, \quad \boldsymbol{r}_{e i 2}=\left(\boldsymbol{r}_{i 2}-d\right) / b$ and obtain $v_{e i 1}=v_{i 1} / a, v_{e i 2}=v_{i 2} / b$ by simple calculations, where $r_{i}=r_{i 1}+j r_{i 2}, v_{i}=v_{i 1}+j v_{i 2}, \quad r_{0}=r_{01}+j r_{02}$, $v_{0}=v_{01}+j v_{02}$.

We can get definition 2 according to the mapping relation.

Definition 2. The leader-following multi-agent system 1 and 2 achieves rotating consensus in an elliptical orbit if

$$
\begin{aligned}
& \lim _{t \rightarrow \infty}\left[\boldsymbol{r}_{i}(t)-\boldsymbol{r}_{0}(t)\right]=0 \\
& \lim _{t \rightarrow \infty}\left[\boldsymbol{r}_{i}(t)-\boldsymbol{r}_{k}(t)\right]=0 \\
& \lim _{t \rightarrow \infty}\left[\boldsymbol{v}_{i}(t)-\boldsymbol{v}_{0}(t)\right]=0 \\
& \lim _{t \rightarrow \infty}\left[\boldsymbol{v}_{i}(t)-\boldsymbol{v}_{\boldsymbol{k}}(t)\right]=0 \\
& \lim _{t \rightarrow \infty}\left[\frac{\dot{\boldsymbol{v}}_{01}(t)}{a}+\boldsymbol{j} \frac{\dot{\boldsymbol{v}}_{02}(t)}{b}-\boldsymbol{j} \omega\left(\frac{\boldsymbol{v}_{01}(t)}{a}+\boldsymbol{j} \frac{\boldsymbol{v}_{02}(t)}{b}\right)\right]=0 \\
& \lim _{t \rightarrow \infty}\left[\frac{\dot{\boldsymbol{v}}_{i 1}(t)}{a}+\boldsymbol{j} \frac{\dot{\boldsymbol{v}}_{i 2}(t)}{b}-\boldsymbol{j} \boldsymbol{\omega}\left(\frac{\boldsymbol{v}_{i 1}(t)}{a}+\boldsymbol{j} \frac{\boldsymbol{v}_{i 2}(t)}{b}\right)\right]=0
\end{aligned}
$$

for all $i, k \in I$.

The position and velocity of all following agents achieve consensus with the leader and surround a common point in an elliptical orbit. Then, this paper will only discuss the rotating consensus of Definition2.

\section{Rotating CONSENSUS}

We assumed $\boldsymbol{\omega}=1$ and used the following consensus algorithm.

A Zero communication time-delay

$$
u_{i}=u_{i 1}+u_{i 2}
$$

where $u_{i 1}=\boldsymbol{j}\left(\frac{\boldsymbol{v}_{i 1}}{a}+\boldsymbol{j} \frac{\boldsymbol{v}_{i 2}}{b}\right)$ and

$$
\begin{aligned}
& u_{i 2}=-k_{1}\left\{\sum_{s_{k} \in N_{i}} a_{i j}\left[\left(\frac{v_{i 1}}{a}+j \frac{v_{i 2}}{b}\right)-\left(\frac{v_{k 1}}{a}+j \frac{v_{k 2}}{b}\right)\right]+a_{i 0}\left[\left(\frac{v_{i 1}}{a}+j \frac{v_{i 2}}{b}\right)\right.\right. \\
& \left.\left.-\left(\frac{v_{01}}{a}+j \frac{v_{02}}{b}\right)\right]\right\}-k_{2}\left\{\sum _ { s _ { k } \in N _ { i } } a _ { i j } \left\{\left[\left(\frac{r_{i 1}-c}{a}+j \frac{r_{i 2}-d}{b}\right)+j\left(\frac{v_{i 1}}{a}+j \frac{v_{i 2}}{b}\right)\right]\right.\right. \\
& \left.-\left[\left(\frac{\boldsymbol{r}_{k 1}-c}{a}+\boldsymbol{j} \frac{\boldsymbol{r}_{k 2}-d}{b}\right)+\boldsymbol{j}\left(\frac{\boldsymbol{v}_{\boldsymbol{k}}}{a}+\boldsymbol{j} \frac{\boldsymbol{v}_{k 2}}{b}\right)\right]\right\}+a_{i 0}\left\{\left[\left(\frac{\boldsymbol{r}_{i 1}-c}{a}+\boldsymbol{j} \frac{\boldsymbol{r}_{i 2}-d}{b}\right)\right.\right. \\
& \left.\left.\left.+\boldsymbol{j}\left(\frac{\boldsymbol{v}_{i 1}}{a}+\boldsymbol{j} \frac{\boldsymbol{v}_{i 2}}{b}\right)\right]-\left[\left(\frac{\boldsymbol{r}_{01}-c}{a}+\boldsymbol{j} \frac{\boldsymbol{r}_{02}-d}{b}\right)+\boldsymbol{j}\left(\frac{\boldsymbol{v}_{01}}{a}+\boldsymbol{j} \frac{\boldsymbol{v}_{02}}{b}\right)\right]\right\}\right\}
\end{aligned}
$$


B Multiple communication time-delays

$$
u_{i}=u_{i 1}+u_{i 2}
$$

where $u_{i 1}=\boldsymbol{j}\left(\frac{\boldsymbol{v}_{i 1}}{a}+\boldsymbol{j} \frac{\boldsymbol{v}_{i 2}}{b}\right)$ and

$$
\begin{aligned}
& u_{i 2}=-k_{1}\left(\sum_{s_{k} \in N_{i}} a_{i j}\left[\left(\frac{v_{i l}\left(t-\tau_{i k}\right)}{a}+j \frac{\boldsymbol{v}_{i 2}\left(t-\tau_{i k}\right)}{b}\right)-\left(\frac{\boldsymbol{v}_{k 1}\left(t-\tau_{i k}\right)}{a}+\boldsymbol{j} \frac{\boldsymbol{v}_{k 2}\left(t-\tau_{i k}\right)}{b}\right)\right]\right. \\
& \left.+a_{i 0}\left[\left(\frac{\boldsymbol{v}_{i 1}\left(t-\tau_{i 0}\right)}{a}+\boldsymbol{j} \frac{\boldsymbol{v}_{i 2}\left(t-\tau_{i 0}\right)}{b}\right)-\left(\frac{\boldsymbol{v}_{01}\left(t-\tau_{i 0}\right)}{a}+\boldsymbol{j} \frac{\boldsymbol{v}_{02}\left(t-\tau_{i 0}\right)}{b}\right)\right]\right\}-k_{2}\left\{\sum_{s_{k} \in N_{i}} a_{i j}\right. \\
& \left\{\left[\left(\frac{\boldsymbol{r}_{i 1}\left(t-\tau_{i k}\right)-c}{a}+\boldsymbol{j} \frac{\boldsymbol{r}_{i 2}\left(t-\tau_{i k}\right)-d}{b}\right)+\boldsymbol{j}\left(\frac{\boldsymbol{v}_{i l}\left(t-\tau_{i k}\right)}{a}+\boldsymbol{j} \frac{\boldsymbol{v}_{i 2}\left(t-\tau_{i k}\right)}{b}\right)\right]\right.
\end{aligned}
$$$$
\left.-\left[\left(\frac{\boldsymbol{r}_{k 1}\left(t-\tau_{i k}\right)-c}{a}+\boldsymbol{j} \frac{\boldsymbol{r}_{k 2}\left(t-\tau_{i k}\right)-d}{b}\right)+\boldsymbol{j}\left(\frac{\boldsymbol{v}_{k 1}\left(t-\tau_{i k}\right)}{a}+\boldsymbol{j} \frac{\boldsymbol{v}_{k 2}\left(t-\tau_{i k}\right)}{b}\right)\right]\right\}
$$$$
+a_{i 0}\left\{\left[\left(\frac{\boldsymbol{r}_{i 1}\left(t-\tau_{i 0}\right)-c}{a}+j \frac{\boldsymbol{r}_{i 2}\left(t-\tau_{i 0}\right)-d}{b}\right)+j\left(\frac{\boldsymbol{v}_{i 1}\left(t-\tau_{i 0}\right)}{a}+j \frac{\boldsymbol{v}_{i 2}\left(t-\tau_{i 0}\right)}{b}\right)\right]\right.
$$$$
\left.\left.-\left[\left(\frac{\boldsymbol{r}_{01}\left(t-\tau_{i 0}\right)-c}{a}+\boldsymbol{j} \frac{\boldsymbol{r}_{02}\left(t-\tau_{i 0}\right)-d}{b}\right)+\boldsymbol{j}\left(\frac{\boldsymbol{v}_{01}\left(t-\tau_{i 0}\right)}{a}+\boldsymbol{j} \frac{\boldsymbol{v}_{02}\left(t-\tau_{i 0}\right)}{b}\right)\right]\right\}\right\}
$$

where $\tau_{i k}$ are the communication time-delays between agent $i$ and its neighbor agent $k$ and satisfy $\tau_{i k}=\tau_{k i}$, $\forall i \neq k$, while $\tau_{i 0}$ denote the communication time-delays between agent $i$ and the leader (labeled 0 ).

Denote $\Phi(t)=\left[\boldsymbol{\xi}_{1}, \boldsymbol{\eta}_{1}, \boldsymbol{\xi}_{2}, \boldsymbol{\eta}_{2}, \cdots, \boldsymbol{\xi}_{n}, \boldsymbol{\eta}_{n}\right]^{T}, \boldsymbol{A}=\left[\begin{array}{cc}\boldsymbol{j} & -\boldsymbol{j} \\ \boldsymbol{0} & \boldsymbol{0}\end{array}\right] \mathrm{a}$ nd $\quad \boldsymbol{B}=\left[\begin{array}{cc}\boldsymbol{0} & \boldsymbol{0} \\ -\boldsymbol{k}_{1} & \boldsymbol{k}_{\boldsymbol{1}}+\boldsymbol{j} \boldsymbol{k}_{2}\end{array}\right], \quad \forall i \in I$ and $k_{1} 、 k_{2} \in R^{n \times n}>0$, where $\xi_{i}$ and $\eta_{i}$ are respectively $\xi_{i}=\left(\frac{\boldsymbol{r}_{i 1}-c}{a}+\boldsymbol{j} \frac{\boldsymbol{r}_{\boldsymbol{i 2}}-d}{b}\right)-\left(\frac{\boldsymbol{r}_{01}-c}{a}+\boldsymbol{j} \frac{\boldsymbol{r}_{02}-d}{b}\right) \quad$ and $\eta_{i}=\left[\left(\frac{\boldsymbol{r}_{i 1}-c}{a}+\boldsymbol{j} \frac{\boldsymbol{r}_{i 2}-d}{b}\right)+\boldsymbol{j}\left(\frac{\boldsymbol{v}_{i 1}}{a}+\boldsymbol{j} \frac{\boldsymbol{v}_{i 2}}{b}\right)\right]-\left[\left(\frac{\boldsymbol{r}_{01}-c}{a}+\boldsymbol{j} \frac{\boldsymbol{r}_{02}-d}{b}\right)+\boldsymbol{j}\left(\frac{\boldsymbol{v}_{01}}{a}+\boldsymbol{j} \frac{\boldsymbol{v}_{02}}{b}\right)\right]$ Then using the protocol (6), the closed-loop network dynamics can be written as

$$
\dot{\Phi}(t)=\left(\boldsymbol{I}_{n} \otimes \boldsymbol{A}-\tilde{\boldsymbol{L}}_{\sigma} \otimes \boldsymbol{B}\right) \Phi(t)
$$

Where, $\tilde{\boldsymbol{L}}_{\sigma}=\boldsymbol{L}_{\sigma}+\boldsymbol{D}$ and $\sigma$ represents switching topology. Similarly, using protocol (7) the network dynamics is

$$
\dot{\Phi}(t)=\left(\boldsymbol{I}_{n} \otimes \boldsymbol{A}\right) \Phi(t)-\left(\tilde{\boldsymbol{L}}_{\sigma} \otimes \boldsymbol{B}\right) \Phi\left(t-\tau_{m}\right)
$$

where $\tau_{m} \in\left\{\tau_{i k}: i, k=1,2 \cdots, n\right\}$ and since the graph $\tilde{G}$ is undirected, we have $\tilde{\boldsymbol{L}}_{\sigma} \boldsymbol{1}=0$ by Lemma 1 . Besides, $\tilde{\boldsymbol{L}}_{\sigma}=\boldsymbol{L}_{\sigma}+\boldsymbol{D}$,then, $\tilde{\boldsymbol{L}}_{\sigma} \boldsymbol{I}=\left[a_{10}, a_{20}, \cdots, a_{\mathrm{n} 0}\right]$.Thus,

$$
\begin{array}{lr}
\boldsymbol{I}^{T}\left[\left(\boldsymbol{I}_{n} \otimes \boldsymbol{A}-\tilde{\boldsymbol{L}}_{\sigma} \otimes \boldsymbol{B}\right) \Phi(t)\right]=\boldsymbol{0} & \text { and } \\
{\left[\left(\boldsymbol{I}_{\boldsymbol{n}} \otimes \boldsymbol{A}\right) \Phi(t)-(L \otimes \boldsymbol{B}) \Phi\left(t-\tau_{i j}\right)\right] \boldsymbol{I}=\mathbf{0}} & \boldsymbol{I}^{T}\left(\tilde{\boldsymbol{L}}_{\sigma}\right.
\end{array}
$$$$
\left.\otimes \boldsymbol{B}) \Phi\left(t-\tau_{m}\right)\right]=\mathbf{0} \text {, i.e., } \sum_{i=1}^{n}\left(\dot{\boldsymbol{\xi}}_{i}(t)+\dot{\boldsymbol{\eta}}_{i}(t)\right)=0 \text {, }
$$

which implies $\alpha=\frac{1}{2 n} \sum_{i=1}^{n}\left(\xi_{i}(t)+\eta_{i}(t)\right)=0$ is an invariant quantity. Let $\delta(t)=\boldsymbol{\Phi}(t)-\alpha 1$. Then the system (8) and (9) can be respectively transformed into

$$
\begin{gathered}
\dot{\delta}(t)=\left(\boldsymbol{I}_{n} \otimes \boldsymbol{A}-\tilde{\boldsymbol{L}}_{\sigma} \otimes \boldsymbol{B}\right) \delta(t) \\
\dot{\boldsymbol{\delta}}(t)=\left(\boldsymbol{I}_{n} \otimes \boldsymbol{A}\right) \delta(t)-\left(\tilde{\boldsymbol{L}}_{\sigma} \otimes \boldsymbol{B}\right) \boldsymbol{\delta}\left(t-\tau_{m}\right) \\
\text { V. STABILITY ANALYSIS }
\end{gathered}
$$

In this section, we divided two cases to study the efficient of the protocols: 1) networks with switching topology and zero communication time-delay; 2) networks with switching topology and multiple time-delays.

\section{A Networks with zero time-delay}

Theorem 1 Consider a network with switching topology that is kept connected. Given the protocol (10), consensus can be achieved if there exist a positive definite Hermite matrix $P \in C^{2 n \times 2 n}$ such that

$$
\left(I_{n} \otimes A-\tilde{L}_{\sigma} \otimes B\right)^{T} P+P\left(I_{n} \otimes A-\tilde{L}_{\sigma} \otimes B\right)<0
$$

Proof: Define a common Lyapunov function for system (10) as $V(t)=\delta^{T}(t) P \delta(t)$, where $P \in C^{2 n \times 2 n}$ is a positive Hermite matrice and $\operatorname{rank}(P)=2 n-2$.Calculating $\dot{V}$ along the trajectories of (10), we have

$$
\begin{aligned}
\dot{V}(t) & =\dot{\delta}^{T}(t) P \delta(t)+\delta^{T}(t) P \dot{\delta}(t) \\
& =\left[\left(I_{n} \otimes A-\tilde{L}_{\sigma} \otimes B\right) \delta(t)\right]^{T} P \delta(t)+\delta^{T}(t) P\left(I_{n} \otimes A-\tilde{L}_{\sigma} \otimes B\right) \delta(t) \\
& =\delta^{T}(t)\left(I_{n} \otimes A-\tilde{L}_{\sigma} \otimes B\right)^{T} P \delta(t)+\delta^{T}(t) P\left(I_{n} \otimes A-\tilde{L}_{\sigma} \otimes B\right) \delta(t) \\
& =\delta^{T}(t)\left[\left(I_{n} \otimes A-\tilde{L}_{\sigma} \otimes B\right)^{T} P+P\left(I_{n} \otimes A-\tilde{L}_{\sigma} \otimes B\right)\right] \delta(t)<0
\end{aligned}
$$

By Lyapunov theory, we have $\lim _{t \rightarrow \infty} \delta(t)=0$. Then, the multi-agent system without time-delay achieve rotating consensus control in elliptical orbit.

\section{B Networks with multiple time-delays}

Theorem 2. Consider a network of second-order agents with multiple time-delays and switching topology that is kept connected. Then the protocol (11) solves the consensus problem, if there exist symmetric positive Hermite matrices $P, Q_{m}, R_{m} \in C^{2 n \times 2 n}$ satisfying

$$
H=\left[\begin{array}{lll}
H_{11} & H_{12} & H_{13} \\
H_{12}{ }^{\mathrm{T}} & H_{22} & 0 \\
H_{13}{ }^{\mathrm{T}} & 0 & H_{33}
\end{array}\right]<0
$$

where

$$
\begin{aligned}
& H_{11}=P\left(I_{n} \otimes A-\tilde{L}_{\sigma} \otimes B\right)+\left(I_{n} \otimes A-\tilde{L}_{\sigma} \otimes B\right)^{T} P+\sum_{m=1}^{M} Q_{m} \\
& +\sum_{m=1}^{M} \tau_{m}\left(I_{n} \otimes A\right)^{T} R_{m}\left(I_{n} \otimes A\right)
\end{aligned}
$$




$$
\begin{gathered}
H_{12}=-\sum_{m=1}^{M} \tau_{m}\left(I_{n} \otimes A\right)^{T} R_{m}\left[\tilde{L}_{\sigma 1} \otimes B, \tilde{L}_{\sigma 2} \otimes B, \cdots, \tilde{L}_{\sigma M} \otimes B\right] \\
H_{13}=\left[P\left(\tilde{L}_{\sigma 1} \otimes B\right), P\left(\tilde{L}_{\sigma 2} \otimes B\right), \cdots, P\left(\tilde{L}_{\sigma M} \otimes B\right)\right], \\
H_{22}=-\operatorname{diag}\left\{Q_{1}, Q_{2}, \cdots, Q_{M}\right\}+\sum_{m=1}^{M} \tau_{m}\left[\tilde{L}_{\sigma 1} \otimes B, \tilde{L}_{\sigma 2} \otimes B, \cdots, \tilde{L}_{\sigma M} \otimes B\right]^{T} \\
R_{m}\left[\tilde{L}_{\sigma 1} \otimes B, \tilde{L}_{\sigma 2} \otimes B, \cdots, \tilde{L}_{\sigma M} \otimes B\right] \\
H_{33}=-\operatorname{diag}\left\{R_{1} / \tau_{1}, R_{2} / \tau_{2}, \cdots, R_{M} / \tau_{m}\right\} .
\end{gathered}
$$

Proof: Define a common Lyapunov function for system (11) as follows

$V(t)=\delta^{T}(t) P \delta(t)+\sum_{m=1}^{M} \int_{t-\tau_{m}}^{t} \delta^{T}(s) Q_{m} \delta(s) d s+\sum_{m=1}^{M} \int_{-\tau_{m}}^{0} \int_{t+\theta}^{t} \dot{\delta}^{T}(s) R_{m} \dot{\delta}(s) d s d \theta$

where, $R_{m} \in C^{2 n \times 2 n}$ is a positive definite matrix, $P, Q_{m} \in C^{2 n \times 2 n}$ are positive Hermite matrices and $\operatorname{rank}(P) \quad=\operatorname{rank}\left(Q_{m}\right)=2 n-2 \quad$ for $i=1,2, \cdots, M$.Calculating $\dot{V}$ along the trajectories of (8), we get

$$
\begin{aligned}
& \dot{V}(t) \leq 2 \delta^{T}(t) P\left(I_{n} \otimes A\right) \delta(t)-2 \delta^{T}(t) P \sum_{m=1}^{M}\left(\tilde{L}_{\sigma m} \otimes B\right) \delta\left(t-\tau_{m}\right)+\sum_{m=1}^{M} \delta^{T}(t) Q_{m} \delta(t) \\
& -\sum_{m=1}^{M} \delta^{T}\left(t-\tau_{m}\right) Q_{m} \delta\left(t-\tau_{m}\right)+\sum_{m=1}^{M} \tau_{m} \dot{\delta}(t)^{T} R_{m} \dot{\delta}(t)-\sum_{m=1}^{M} \int_{t-\tau_{m}}^{t} \dot{\delta}^{T}(s) R_{m} \dot{\delta}(s) d s
\end{aligned}
$$

Then by Newton-Leibniz formula and for any vector $x, y \in \boldsymbol{C}^{n}$ and symmetric positive definite Hermite matrix

$$
\begin{aligned}
& \wp \in C^{n \times n}, 2 x^{T} y \leq x^{T} \wp^{-1} x+y^{T} \wp y \\
& \quad \text { we have } \\
& \quad-2 \delta^{T}(t) P\left(\tilde{L}_{\sigma m} \otimes B\right) \delta\left(t-\tau_{m}\right)=-2 \delta^{T}(t) P\left(\tilde{L}_{\sigma m} \otimes B\right) \delta(t) \\
& +\tau_{m} \delta^{T}(t) P\left(\tilde{L}_{\sigma m} \otimes B\right) R_{m}^{-1}\left(\tilde{L}_{\sigma m} \otimes B\right)^{T} P \delta(t)+\sum_{m=1}^{M} \int_{t-\tau_{m}}^{t} \dot{\delta}^{T}(s) R_{m} \dot{\delta}(s) d s \\
& \quad+\int_{t-\tau_{m}}^{t} 2 \delta^{T}(t) P\left(\tilde{L}_{\sigma m} \otimes B\right) \dot{\delta}(s) d s \leq-2 \delta^{T}(t) P\left(\tilde{L}_{\sigma m} \otimes B\right) \delta(t)
\end{aligned}
$$

where $m=1,2 \cdots, M$ And since $\sum_{m=1}^{n} \tilde{L}_{\sigma m}=\tilde{L}_{\sigma}$, we have

$\dot{V}(t) \leq 2 \delta^{T}(t) P\left(I_{n} \otimes A-\tilde{L}_{\sigma} \otimes B\right) \delta(t)+\sum_{m=1}^{M} \delta^{T}(t) Q_{m} \delta(t)-\sum_{m=1}^{M} \delta^{T}\left(t-\tau_{m}\right) Q_{m} \delta\left(t-\tau_{m}\right)$

$+\sum_{m=1}^{M} \tau_{m} T^{T}(t) P\left(\tilde{L}_{\sigma m} \otimes B\right) R_{m}^{-1}\left(\tilde{L}_{\sigma m} \otimes B\right)^{T} P \delta(t)+\sum_{m=1}^{M} \tau_{m}\left[\left(I_{n} \otimes A\right) \delta(t)-\left(\tilde{L}_{\sigma m} \otimes B\right) \delta\left(t-\tau_{m}\right)\right]^{T}$

$R_{m}\left[\left(I_{n} \otimes A\right) \delta(t)-\left(\tilde{L}_{\sigma m} \otimes B\right) \delta\left(t-\tau_{m}\right)\right]=\eta^{T}(t)\left[\begin{array}{ll}\bar{H}_{11} & H_{12} \\ H_{12}^{T} & H_{22}\end{array}\right] \eta(t)$

where $\eta^{T}(t)=\left[\delta^{T}(t), \delta^{T}\left(t-\tau_{1}\right), \delta^{T}\left(t-\tau_{2}\right), \cdots, \delta^{T}\left(t-\tau_{M}\right)\right]$, $\bar{H}_{11}=P\left(I_{n} \otimes A-\tilde{L}_{\sigma} \otimes B\right)+\left(I_{n} \otimes A-\tilde{L}_{\sigma} \otimes B\right)^{T} P+\sum_{m=1}^{M} Q_{m}+\sum_{m=1}^{M} \tau_{m}\left(I_{n} \otimes A\right)^{T} R_{m}\left(I_{n} \otimes A\right)$

$+\sum_{m=1}^{M} \tau_{m} P\left[\tilde{L}_{\sigma 1} \otimes B, \tilde{L}_{\sigma 2} \otimes B, \cdots, \tilde{L}_{\sigma M} \otimes B\right] R_{m}^{-1}\left[\tilde{L}_{\sigma 1} \otimes B, \tilde{L}_{\sigma 2} \otimes B, \cdots, \tilde{L}_{\sigma M} \otimes B\right]^{T} P$

$$
\begin{aligned}
& H_{12}=-\sum_{m=1}^{M} \tau_{m}\left(I_{n} \otimes A\right)^{T} R_{m}\left[\tilde{L}_{\sigma 1} \otimes B, \tilde{L}_{\sigma 2} \otimes B, \cdots, \tilde{L}_{\sigma M} \otimes B\right] \\
& H_{22}=-\operatorname{diag}\left\{Q_{1}, Q_{2}, \cdots, Q_{M}\right\}+\sum_{m=1}^{M} \tau_{m}\left[\tilde{L}_{\sigma 1} \otimes B, \tilde{L}_{\sigma 2} \otimes B, \cdots, \tilde{L}_{\sigma M} \otimes B\right]^{T} R_{m} \\
& {\left[\tilde{L}_{\sigma 1} \otimes B, \tilde{L}_{\sigma 2} \otimes B, \cdots, \tilde{L}_{\sigma M} \otimes B\right]}
\end{aligned}
$$

Then, a sufficient condition for $\dot{V}(t)<0$ is that

$$
\bar{H}=\left[\begin{array}{ll}
\bar{H}_{11} & H_{12} \\
H_{12}^{T} & H_{22}
\end{array}\right]<0
$$

Further, by Lemma 2, $\bar{H}<0$ holds if and only if $H<0$.Then, we have $\lim _{t \rightarrow \infty} \delta(t)=0$ and the leader-following multi-agent system with multiple time-delays achieves rotating consensus in an elliptical orbit.

\section{SIMULATION}

Consider a second-order multi-agent system with four agents and one leader. Suppose that the weight of each edge is 1 . Now, we present simulation results for the cases of switching topology with and without time-delay. Figure. 1 shows the communication topology which is switched from one topology to another one every $1 \mathrm{~s}$ in the sequence of $\left[\tilde{G}_{a}, \tilde{G}_{b}, \tilde{G}_{c}, \tilde{G}_{d}, \tilde{G}_{a}\right]$.Fig. 2 shows the position trajectories of the leader following multi-agent using protocol (6) without time delay. Fig. 3 and 4 show the position trajectories of the leader-following multi-agent using protocol (7) respectively with $\tau=\left[\begin{array}{lllllllllll}0.15 & 0.15 & 0.1 & 0.1 & 0.15 & 0.15 & 0.1 & 0.1 & 0.2 & 0.2 & 0.2\end{array}\right.$

$0.20 .10 .10 .10 .1]$

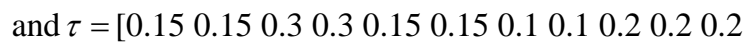

$\left.\begin{array}{lllll}0.2 & 0.1 & 0.1 & 0.3 & 0.3\end{array}\right]$

We can see that Fig. 2 and 3 achieve leader-following rotating consensus in an elliptical orbit. However, the system will diverge when the time-delay is not small enough such as the case in Fig.4. We need further research to confirm a specific time-delays.

\section{CONCLUSIONS}

This paper investigated rotating consensus of second-order leader-following multi-agent system with switching topology and with and without time-delays in an elliptical orbit. We define consensus problem of leader-following multi-agent and propose rotating consensus protocols in two cases. In each case, we present a condition under which all leader-following multi-agent achieve rotating consensus in an elliptical orbit. Simulation results are provided to demonstrate the effectiveness of our theoretical results.

\section{REFERENCES}

[1] Gang Chen, Frank L. Lewis. Leader-following control for multiple inertial agents. Int. J. Robust. Nonlinear Control ,2011,21:925-942

[2] Hong Y G, Chen G R, Linda B. Distributed observers design for leader-following control of multi-agent networks[J]. Automatica, 2008, $44: 846-850$.

[3] Jiangping $\mathrm{Hu}$, Yiguang Hong . Leader-following coordination of multi-agent systems with coupling time delays. Physica A,2007,374:853-863

[4] Zhao, Huanyu, Xu, Shengyuan and Yuan, Deming. Consensus of data-sampled multi-agent systems with Markovian switching topologies[J]. Asian Journal of CONTROL,2012,14(5): 1366-1373.

[5] Liu, Zhi-Wei, Guan, Zhi-Hong, Li, Tao, Zhang, Xian-He and Xiao, Jiang-Wen. QUANTIZED CONSENSUS OF MULTI-AGENT 
SYSTEMS VIA BROADCAST GOSSIP ALGORITHMS[J]. ASIAN JOURNAL OF CONTROL,2012,14(6): 1634-1642.

[6] Sepulchre R, Paley D, Leonard NE. Stabilization of planar collective motion with limited communication[J]. IEEE Transactions on Automatic Control, 2008,53(3):706-719.

[7] Pavone M, Frazzoli E. Decentralized policies for geometric pattern formation and path coverage[J]. ASME Journal of Dynamic Systems, Measurement, and Control, 2007 ,129(5): 633-643.

[8] Lin P, Jia Y. Distributed rotating formation control of multi-agent systems[J]. Systems and Control Letters 2010,59(10):587-595.

[9] Ren W. Collective motion from consensus with Cartesian coordinate coupling[J]. IEEE Transactions on Automatic Control, 2009,54(6): 1330-1335.

[10] YANG Tan, WANG Wenyong, HUANG Lishen. Multi-agent Rotating Consensus Using Only Local Position Information in Three-dimensional Space. in Proceedings of the 30th Chinese Control Conference,2011:4787-4791.

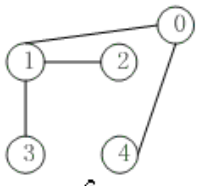

G.

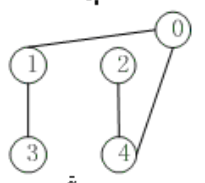

$\bar{G}$.

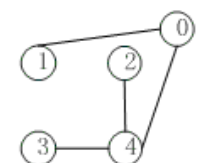

$G_{b}^{*}$

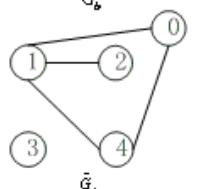

Fig.1. Network topology.

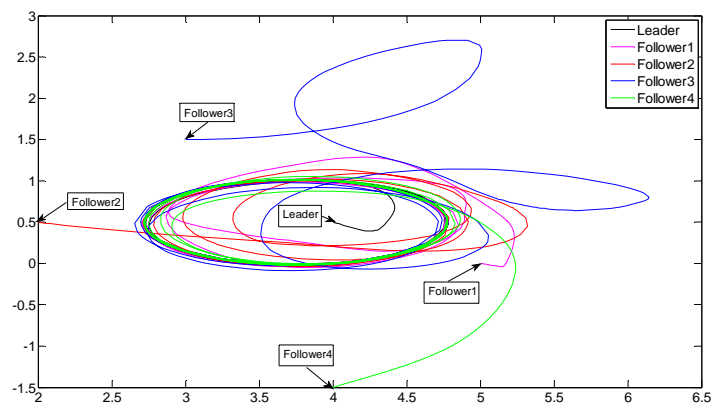

Fig.2. Position trajectories without time-delay.

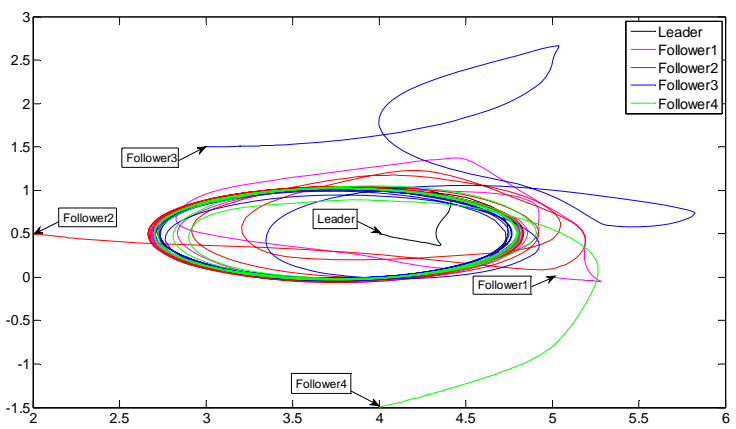

Fig.3. Position trajectories with multiple time-delays.

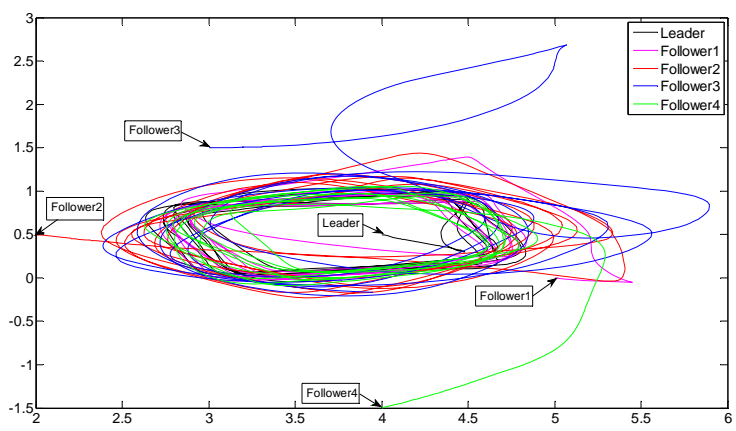

Fig.4. Position trajectories with multiple time-delays. 